\section{Leishmaniose tegumentar americana em crianças: aspectos epidemiológicos de casos atendidos em Manaus, Amazonas, Brasil}

\author{
American tegumentary leishmaniasis in children: \\ epidemiological aspects of cases treated in \\ Manaus, Amazonas, Brazil
}

\footnotetext{
${ }^{1}$ Fundação de Medicina Tropical do Amazonas, Manaus, Brasil.

2 Universidade do Estado Amazonas, Manaus, Brasil. 3 Centro Universitário Nilton Lins, Manaus, Brasil. 4 Universidade Federal do Amazonas, Manaus, Brasil. + Falecido.

Correspondência J. A. O. Guerra Gerência de Leishmanioses, Fundação de Medicina Tropical do Amazonas. Av. Pedro Teixeira 25, Manaus, $A M$ 69040-000, Brasil. jorge@fmt.am.gov.br
}

\begin{abstract}
Children from birth to 14 years of age with American tegumentary leishmaniasis were monitored at the Foundation for Tropical Medicine in the State of Amazonas, Brazil, from January to December 2005; 147 cases were recorded, 55.78\% of which were male and $48.3 \%$ from the Rio Preto da Eva district. For 90 patients (67.67\%), parents or guardians were interviewed about housing in the area where the infection was presumably acquired and the child's habits. Of the group, $58.89 \%$ of the children lived in the presumed area of infection, $60 \%$ customarily entered the forest with their parents, $91.11 \%$ lived in households with domestic animals, $77.78 \%$ of the residences were located within 100 meters of the forest; and $76.67 \%$ of the patients had at least one relative with a history of American tegumentary leishmaniasis. The transmission pattern was related to activities in the forest around the houses and the living situation near the primary forest, with cases of American tegumentary leishmaniasis found in very young children, suggesting transmission in and around the house, and in a few cases, children entering the forest.
\end{abstract}

Leishmaniasis; Child; Infection

\author{
Jorge Augusto de Oliveira Guerra 1,2 \\ Maria das Graças Vale Barbosa 1,2,3 \\ Adalgisa Câmara de Sá Peixoto Loureiro ${ }^{+}$ \\ Candisse Pinheiro Coelho 4 \\ Gilmar Garcias Rosa 4 \\ Leila Inês de Aguiar da Câmara Raposo Coelho 1,4
}

\section{Introdução}

A leishmaniose é considerada pela Organização Mundial da Saúde como uma das cinco doenças infecto-parasitárias endêmicas de maior relevância e um problema de saúde pública mundial 1 .

O Brasil apresenta importante incremento de casos com um duplo perfil epidemiológico observado, expresso pela manutenção de casos oriundos dos focos antigos ou de áreas próximas a eles e, ainda, pelo aparecimento de surtos epidêmicos associados especialmente a acelerado processo de expansão das fronteiras agrícolas, a implantação de áreas de garimpos, a construção de rodovias e invasões na periferia das cidades 2 .

No período de 1985 a 1999, foram registrados no Brasil 388.155 casos autóctones de leishmaniose tegumentar americana; de 1999 a 2003 registraram-se 33.872 casos de leishmaniose tegumentar americana/ano. A Região Norte apresenta o maior número com 61.339 casos, no período descrito, e taxas de incidência por 100 mil habitantes entre 114,8 a 163,5. No Amazonas, também, de 1999 a 2003, foram registrados 12.005 casos, apresentando taxas médias de incidência de 86,77 , e no ano de 2003 essa taxa foi de 121,03 com 3.174 casos, $60,18 \%$ deles no Município de Manaus (Rede internacional de informações para a saúde. http://tabnet.datasus.gov.br/, acessado em 23/Out/2005).

No Estado do Amazonas, a leishmaniose tegumentar americana é primariamente uma zo- 
onose 3 , em que o ciclo de transmissão ocorre entre os flebotomíneos e os animais silvestres e, na maioria das vezes, o homem se infecta ao alterar o ambiente, interpondo-se no ciclo silvestre ao penetrar nesse ecossistema ${ }^{4}$. A doença ocorre, mais habitualmente, na forma de surtos epidêmicos. Dessa forma, o grau de exposição dos indivíduos acometidos está relacionado diretamente a assentamentos populacionais agrícolas planejados ou mais freqüentemente oriundos de processos de ocupação na periferia da cidade, em sua maioria, desordenadas 5,6,7.

Em decorrência disso, os indivíduos ficam expostos aos vetores 8 , pois grande parte das casas em assentamentos populacionais recentes são construídas muito próximas da orla da floresta e os indivíduos são alcançados pelo raio de ação desses vetores, que chegam às casas também atraídos por diversos fatores como a luz, a presença de animais sinantrópicos como Didelphis marsupialis, animais domésticos e o próprio homem. A despeito disso, a transmissão nessas áreas parece ser transitória, visto que, uma vez completada a urbanização, a transmissão é interrompida 9,10,11.

No período de 1985 a 1997, foram registrados 25.917 casos de leishmaniose tegumentar americana no Estado do Amazonas, sendo 15.221 (58,73\%) dentro do Município de Manaus 11. A Fundação de Medicina Tropical do Amazonas (FMTAM), que atende 47,5\% dos casos de leishmaniose do Estado, vem registrando em média mil casos novos a cada ano, grande parte oriunda do Município de Manaus, particularmente de duas estradas: AM-010, que liga Manaus aos municípios de Rio Preto da Eva e Itacoatiara e BR174, que liga Manaus ao Município de Presidente Figueiredo (Amazonas) e ao Estado de Roraima.

No período de abril de 2002 a setembro de 2003, foram atendidos 642 pacientes menores de 12 anos de idade na FMTAM, centro de referência para atendimento dos pacientes com leishmaniose tegumentar americana, onde o registro da doença em crianças é expressivo; a avaliação de fatores relacionados à ocorrência dessa patologia, portanto, é de grande importância em saúde pública, a fim de se proporem medidas factíveis para o seu controle.

Este estudo teve dessa forma o objetivo de avaliar fatores relacionados à ocorrência de leishmaniose tegumentar americana em crianças atendidas na FMTAM, no período de janeiro a dezembro de 2005.

\section{Metodologia}

Trata-se de um estudo de coorte, prospectivo de casos de leishmaniose tegumentar americana em crianças de 0 a 14 anos de idade, realizado mediante dados coletados da ficha clínica na Gerência de Leishmaniose da FMTAM e de um questionário aplicado aos responsáveis pelas crianças durante o atendimento ambulatorial na FMTAM, no período de janeiro a dezembro de 2005.

As variáveis avaliadas a partir do atendimento ambulatorial e com base nas informações da ficha clínica foram: sexo, idade, distribuição temporal dos casos, endereço e procedência de todas as crianças atendidas. Um questionário aplicado aos responsáveis pelas crianças avaliou especificamente as características do provável local de infecção, presença de animais, distância da casa para a floresta, penetração na floresta, atividade exercida pelo paciente e seus pais no local e existência de outros casos na família.

Foram avaliadas crianças portadoras de leishmaniose tegumentar americana, cujos representantes legais tenham concordado e assinado o Termo de Consentimento Livre e Esclarecido, no período de janeiro a dezembro de 2005.

$\mathrm{Na}$ análise estatística, foi utilizado o teste quiquadrado no nível de $5 \%$ de significância.

Todos os procedimentos necessários ao estudo e relativos aos pacientes foram realizados no Ambulatório de Dermatologia e no Laboratório de Leishmaniose da FMTAM.

O estudo foi aprovado pelo comitê de ética em pesquisas da FMTAM.

\section{Resultados}

Durante o ano de 2005 foram atendidos na FMTAM 712 portadores de leishmaniose tegumentar americana, dos quais 147 (20,65\%) eram crianças de até 14 anos de idade, observando-se maior registro de casos na faixa de idade entre 11 e 14 anos, totalizando 50 casos (Figura 1). Houve predomínio de casos de janeiro a março e de novembro a dezembro, período de maior pluviosidade na região (Figura 2). Entre os pacientes, 82 $(55,78 \%)$ eram do sexo masculino e $65(44,22 \%)$ do sexo feminino.

As formas clínicas encontradas foram predominantemente cutâneas. Um paciente, procedente de Cruzeiro do Sul, no Acre, onde predomina a Leishmania (Vianna) braziliensis, apresentava concomitância de lesões mucosa e cutânea.

Entre os 147 pacientes, nove eram casos de reinfecção, seis deles contraíram a lesão no mes- 
mo local da infecção anterior, e três pacientes não tinham registro do local.

A maioria dos casos, $48,30 \%$, era autóctone do Município de Rio Preto da Eva e 43,54\% eram de Manaus (Figura 3).

Figura 1

Distribuição por faixa etária dos casos de leishmaniose tegumentar americana atendidos na Fundação de Medicina Tropical do Amazonas, de janeiro a dezembro 2005.

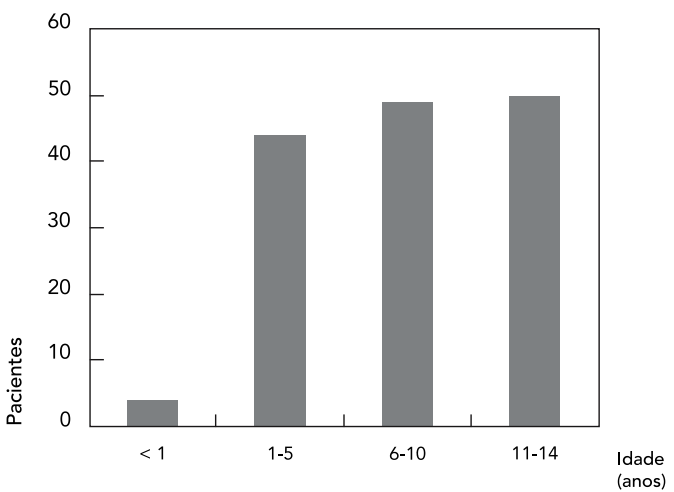

Figura 2

Distribuição anual dos casos de leishmaniose tegumentar americana em crianças, atendidos na Fundação de Medicina Tropical do Amazonas, de janeiro a dezembro de 2005.

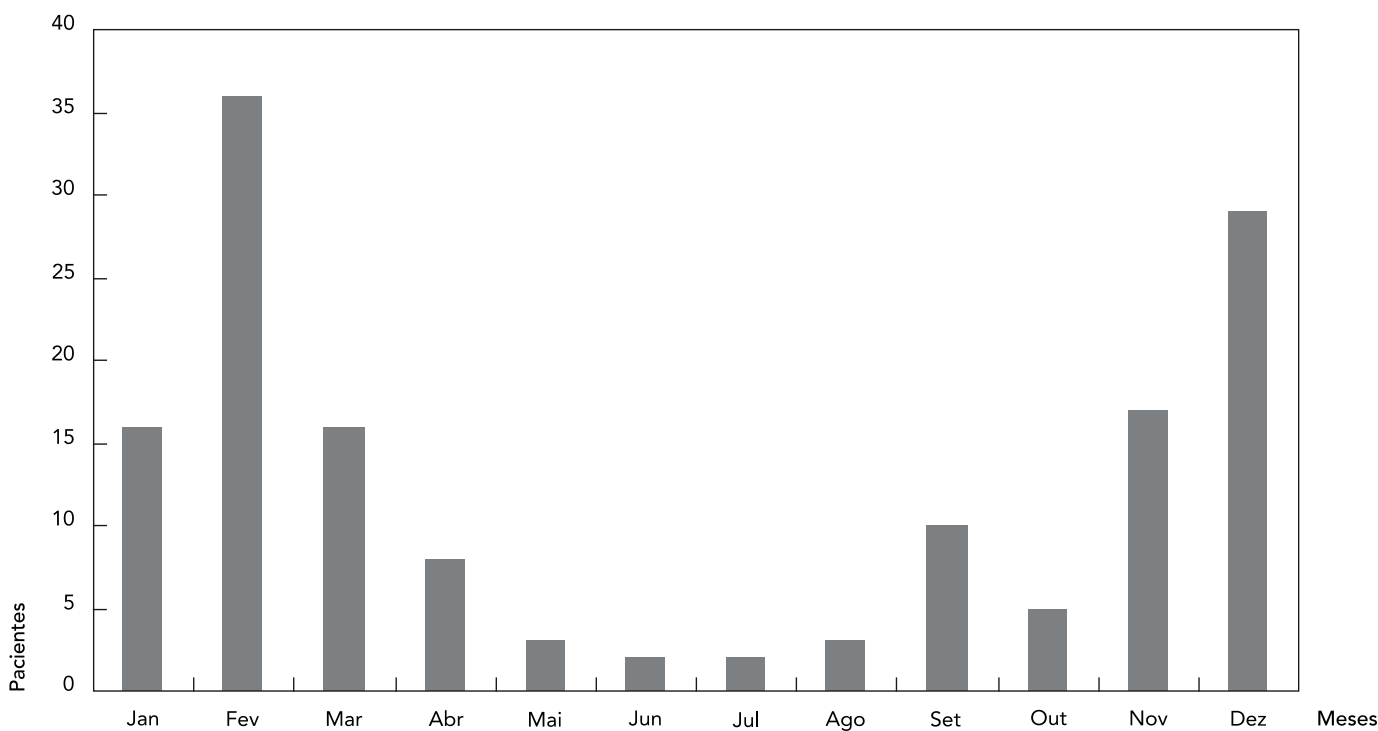

A maior parte das crianças procedia de duas estradas: 76 pacientes $(51,70 \%)$ da AM-010 e 60 pacientes $(40,82 \%)$ da BR-174. Onze casos não eram procedentes dessas estradas. Entre esses, apenas quatro eram da periferia de Manaus.

O questionário foi aplicado a $90(67,67 \%)$ dos responsáveis pelas crianças. Observou-se que 53 (58,89\%) residiam no local da provável infecção e 35 (38,89\%) lá se encontravam por lazer (visita a parentes, férias); $60 \%$ penetravam na mata acompanhando os pais, a maioria em suas atividades de plantio, extrativismo, lazer e obtenção de água e caça.

Em $82(91,11 \%)$ casos, houve relato da presença de animais domésticos, na moradia, no peridomicílio, com diferença significativa $(p<0,05)$. Os animais mais freqüentemente referidos foram cães $(76,67 \%)$, galinhas $(67,78 \%)$ e gatos; os animais silvestres criados ou mantidos em cativeiro foram classificados como "outros" (Figura 4).

Em relação às moradias, 60 (66,67\%) eram de madeira, e registrou-se diferença significativa $(\mathrm{p}<0,05)$ em relação à distância destas para a mata; $77,78 \%$ localizavam-se a até $100 \mathrm{~m}$ da mata (Figura 5). Em 74 casos foi relatada a presença de mata secundária e fragmentos de floresta próximos da casa, além de plantações variadas, inclusos fruteiras, no peridomicílio. 


\section{Figura 3}

Distribuição dos municípios amazonenses de procedência dos casos de leishmaniose tegumentar americana em crianças, atendidos na Fundação de Medicina Tropical do Amazonas, de janeiro a dezembro de 2005.

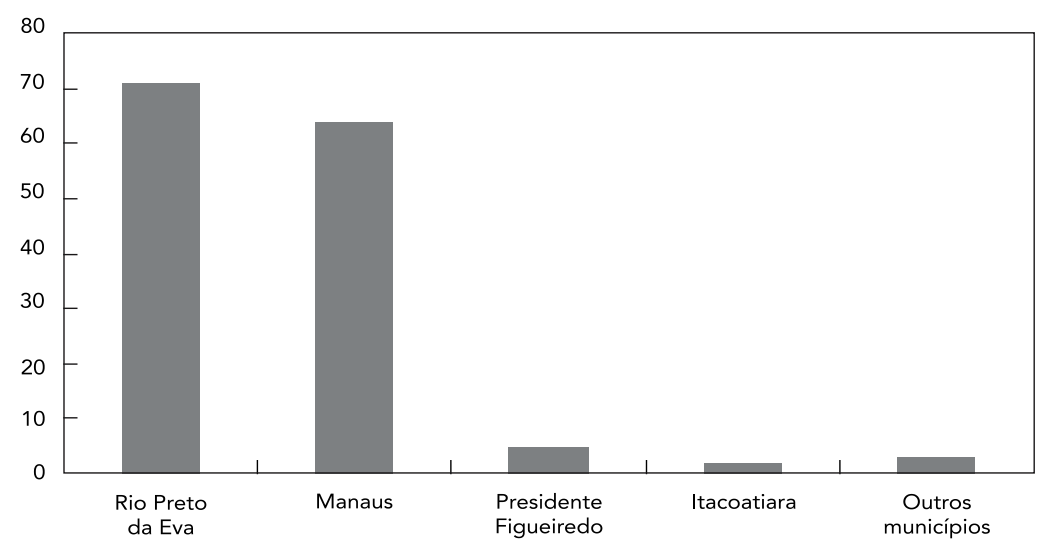

Nota: dois casos são procedentes de outros Estados.
Em 69 (76,67\%) pacientes havia pelo menos um parente com história de leishmaniose tegumentar americana. Houve diferença significativa $(\mathrm{p}<0,05)$ quando se compararam as crianças com e sem história familiar de leishmaniose tegumentar americana. Em 34 casos, os parentes adquiriram a doença no mesmo local e no mesmo período (100 parentes). Em 29 casos os parentes adquiriram a doença no mesmo local, mas em época diferente (60 parentes), e em seis casos os parentes adquiriram a doença em local e época diferentes (8 parentes). Houve uma variação entre 1 e 15 parentes acometidos, sendo a média de 1,85 para cada criança. Algumas crianças tinham parentes em mais de uma das situações.

\section{Discussão}

Em diversas regiões do país, como no Sul e Sudeste, ocorreram intensas modificações ambientais, devido à ação antrópica, atividades agrícolas e

Figura 4

Distribuição dos animais encontrados no peridomicílio de ocorrência dos casos de leishmaniose tegumentar americana em crianças atendidos na Fundação de Medicina Tropical do Amazonas, de janeiro a dezembro de 2005.

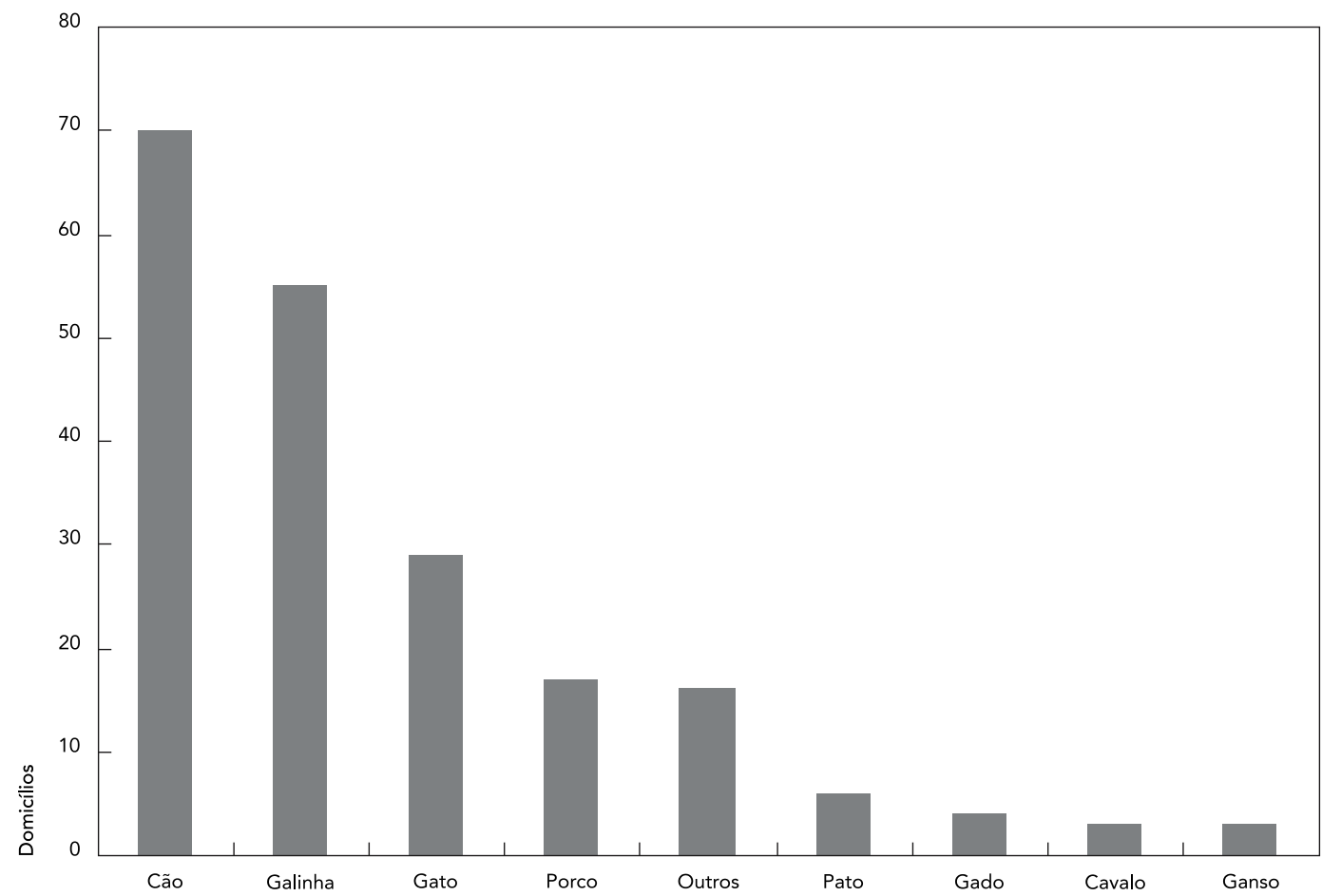


Distância do domicílio à mata, dos casos de leishmaniose tegumentar americana em crianças atendidas na Fundação de Medicina Tropical do Amazonas, de janeiro a dezembro 2005.

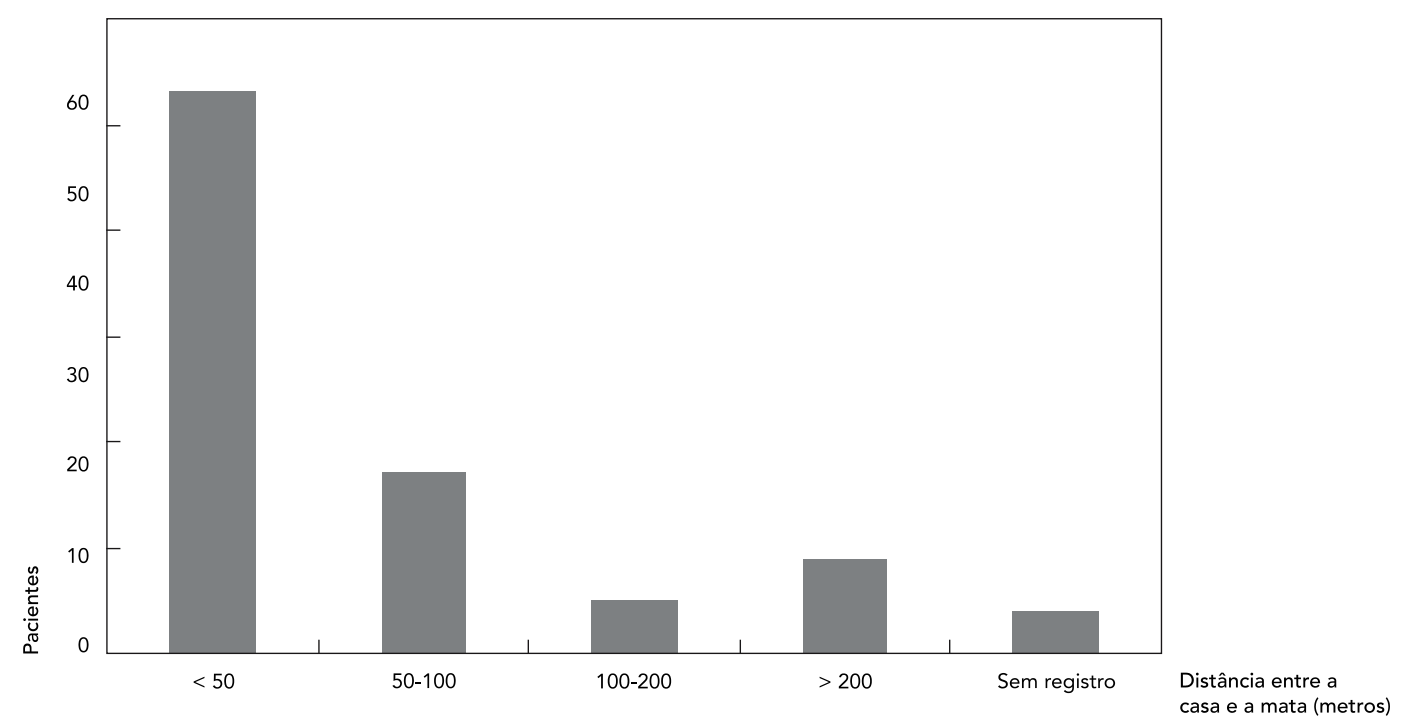

pastoril, levando ao quase desaparecimento da leishmaniose cutânea no final da década de 40 12,13. Entretanto, a partir das décadas de 70 e 80 a leishmaniose reaparece, nessas regiões, com aumento significativo do número de casos surgindo de novas áreas endêmicas 14,15.

Tem-se estabelecido a ocorrência da domiciliação gradual de vetores no decorrer do tempo em áreas de antiga colonização humana onde existe pouca floresta primária remanescente, ocorrendo uma adaptação dos diferentes componentes do ciclo de transmissão, inclusive com presença de novos reservatórios secundários que facilitariam esse tipo de transmissão 16. Essas condições ainda não são observadas em Manaus. Contudo, Paes et al. ${ }^{8}$ descrevem um surto de 22 casos de leishmaniose tegumentar americana por reintrodução da transmissão em área urbanizada da cidade de Manaus, onde existe mata adjacente, tendo encontrado 9,8\% dos casos em menores de cinco anos de idade, sugerindo que a transmissão ocorre no intra e peridomicílio, fato comprovado posteriormente, por intermédio da coleta de diversos transmissores no intra e peridomicílio, encontrando-se também cães com sorologia positiva para Leishmania, porém sem evidência de doença 17.

No ano de 2005 foram registrados na FMTAM, para todas as faixas etárias, incluindo adultos e crianças, $25,7 \%$ de casos no sexo feminino e $74,3 \%$ no masculino, sugerindo um perfil geral relacionado à exposição devido ao trabalho, sobretudo quando este envolve atividades na floresta 18 .

Entre as crianças deste trabalho foi observado um predomínio para o sexo masculino, concordando com os achados de outros estudos 12,19. Martins et al. 20, no Município de Buriticupu, Maranhão, observaram que a doença atingiu todas as faixas etárias, com baixa freqüência nos menores de cinco anos, porém, tendendo a aumentar progressivamente, à medida que avançam as faixas etárias, concordando também com os dados deste estudo em que se observou maior ocorrência na faixa de 11 a 14 anos, possivelmente porque já auxiliam seus pais em atividades de agricultura e retirada de madeira para fazer carvão e busca de água nos igarapés.

Segundo Passos et al. 21, o maior número de casos de leishmaniose tegumentar americana entre homens e adultos sugere transmissão extradomiciliar em população economicamente ativa, enquanto essa ocorrência entre mulheres, crianças e pessoas com ocupações não agrícolas sugere a transmissão intra e/ou peridomiciliar.

Natal et al. 22, observando a ocorrência da leishmaniose tegumentar americana no Estado do Acre, onde as formas mucosas e cutâneo-mu- 
cosas somam $15,9 \%$ dos casos totais da região, encontraram $25 \%$ de ocorrência de leishmaniose tegumentar americana em crianças menores de 15 anos. Neste estudo, foi encontrado o percentual de $20,65 \%$, no entanto, em uma faixa etária de até 14 anos. Quando se somaram a isso os dados de atendimento de casos de leishmaniose tegumentar americana na faixa de 15 anos no ano de 2005 , o percentual total chegou a $22,33 \%$, sendo $42,18 \%$ para a faixa etária de 11 a 15 anos, com 62 casos registrados.

O perfil de transmissão entre menores, especialmente entre aqueles com idade igual ou inferior a cinco anos é sugestivo de contato com o vetor no intra e peridomicílio 23 . Dos 90 pacientes entrevistados neste estudo, $70 \%$ tinham pelo menos um parente com leishmaniose tegumentar americana. Na maior parte, os parentes acometidos adquiriram a doença no mesmo local e no mesmo período de infecção do paciente. Deve-se ressaltar que em determinadas circunstâncias, as mães levam os filhos aos locais de trabalho, particularmente na lavoura, aumentando a exposição dos menores aos vetores; porém, em áreas de transmissão, mais recentes, a doença ocorre em todas as faixas etárias, embora tenda a afetar a criança com alta freqüência 23 , também demonstrado neste estudo, inclusive com casos de reinfecção.

Castro et al. 12, no Estado do Paraná, observaram que fragmentos ou reservas de mata primária entre as plantações favoreciam o aparecimento de pequenos focos de leishmaniose, pois ofereciam condições adequadas ao desenvolvimento dos vetores. Tais autores descrevem ainda que as casas nas zonas rurais são construídas ao lado desses fragmentos e que frutíferas, bambuzais e outras vegetações arbustivas, entre a reserva florestal e as residências, permitem a passagem dos flebotomíneos da reserva ao peridomicílio, possibilitando condições para a transmissão no intra e peridomicílio. De acordo com as informações obtidas neste estudo, a maior parte das casas dos pacientes encontrava-se a uma distancia de até $100 \mathrm{~m}$ da mata, a maioria com plantações de fruteiras e mata secundária nas proximidades, oferecendo as mesmas condições observadas no Paraná.

Sosa-Estani et al. 24 concluíram que entre os riscos de adquirir leishmaniose tegumentar americana no domicilio incluía-se o hábito de dormir fora do quarto, em casas com varandas e sem fechaduras adequadas nas janelas. Nas áreas deste estudo predominam casas de madeira, com muitas frestas, facilitando a inserção dos vetores no domicílio em qualquer horário. Em $41,11 \%$ das crianças deste estudo não havia relatos de contato com a mata, sugerindo a presença do transmissor no peri e intradomicílio, fato reforçado pelo aparecimento de lesões em crianças de zero a um ano de idade que não foram levadas à mata. Também havia relatos de pacientes que habitavam casas sem paredes completas ou dormiam em locais abertos como varandas ou embaixo das árvores no quintal.

A adaptação do flebotomíneo ao ambiente peridomiciliar ou até mesmo domiciliar propicia a transmissão da Leishmania a animais domésticos como cães e eqüinos, assim como ao homem, tornando a probabilidade de transmissão semelhante em toda a população que se encontra sob risco, não importando a faixa etária, sexo ou atividade profissional 25 .

A transmissão da leishmaniose tegumentar americana no Amazonas apresenta uma evidente variação sazonal, sendo mais intensa no período mais chuvoso, quando temperatura, insolação e evaporação estão mais baixas e a umidade mais alta, propiciando aumento da densidade dos flebótomos, incluindo as espécies envolvidas no ciclo da doença ${ }^{13}$. Neste estudo verificou-se um padrão sazonal bem definido na região com maior registro de doentes durante o período chuvoso: meses de dezembro a maio, como já demonstrado em outros estudos 8,10,26. O elevado número de pacientes no mês de fevereiro pode ser atribuído à exposição maior das crianças em férias, nos meses de dezembro e janeiro, seja retornando à casa de seus pais, visto que muitos vão para Manaus estudar em casa de parentes, seja por se encontrarem visitando esses parentes em área rural.

A presença de animais domésticos foi freqüentemente relatada neste estudo, podendo os animais servir como fonte de atração aos vetores, mantendo dessa forma o ciclo de transmissão da leishmaniose tegumentar americana no peridomicílio, como observado em outros estudos 9,16,23. Por outro lado, os locais de criação de aves domésticas, ou com lixo depositado na periferia das casas, como as deste estudo, podem funcionar como fatores de atração para marsupiais, que por sua vez facilitam a manutenção do ciclo de transmissão da leishmaniose no peridomicílio humano ${ }^{9}$. O Didelphis marsupialis adquire especial importância em áreas de floresta alteradas pelo homem, com uma taxa de infecção na região de Manaus de mais de $20 \% 27$. Esse animal se alimenta em refugos domésticos nos limites da floresta próximos às casas 4 , ocorrendo constante circulação entre o meio florestal, onde se infecta, e o ambiente humano, onde serve de fonte de infecção para os flebótomos ali presentes ${ }^{9}$, estabelecendo possivelmente, dessa forma, um elo entre o ciclo silvestre e o peridomicílio 28 . 
A exposição das crianças também tem sido atribuída às atividades dos pais, que também é exercida por esses menores, sobretudo dentro dos assentamentos populacionais ${ }^{29}$. Neste estudo foi observado que a terça parte dos entrevistados referiu que as crianças acompanham seus pais em atividades que as expõem ao risco de adquirir leishmaniose tegumentar americana.

Neste estudo, pode-se, portanto, aventar a possibilidade de transmissão em duas situações distintas: (a) no intra peridomicílio - justificado pelo aparecimento de casos em menores de cinco anos de idade e com animais domésticos provavelmente servindo de fonte de atração aos vetores e ainda pela proximidade das habitações humanas com a mata; (b) na mata - ocorrendo em crianças maiores, por causa das atividades exercidas pelas crianças, com ou sem seus pais, como retirada de madeira para fazer carvão ou uso doméstico, busca de alimentos, como frutas e caça e no auxilio aos pais em atividades agrícolas.

No período de janeiro a dezembro de 2002, as crianças menores de 15 anos de idade representaram 19,84\% dos casos atendidos na FMTAM. Em 2003, foram 21,71\%; em 2004, foram 20,18\%. O maior número de casos ocorre em bairros da zona leste, por conta da expansão da cidade, e ao longo de duas estradas: AM-010 e BR-174, onde respectivamente dois assentamentos populacio-

\section{Resumo}

No período entre janeiro a dezembro de 2005, foram avaliadas crianças de zero a 14 anos de idade com leishmaniose tegumentar americana, atendidas na Fundação de Medicina Tropical do Amazonas; registraram-se 147 casos; 55,78\% do sexo masculino; 48,3\% procediam do Município de Rio Preto da Eva; 76\% procediam de duas estradas. Entre 90 (67,67\%) pacientes, entrevistaram-se os responsáveis sobre a moradia na área de transmissão e os hábitos da criança. Desses, $58,89 \%$ residiam no local da provável infecção; $60 \%$ das crianças penetravam na mata acompanhando os pais; em 91,11\% dos casos havia animais domésticos na moradia; $77,78 \%$ das residências localizavam-se até $100 \mathrm{~m}$ de distância da mata; $76,67 \%$ pacientes tinham pelo menos um parente com história de leishmaniose tegumentar americana. O padrão de transmissão foi relacionado especialmente a atividades de manejo florestal ocorrendo próximas ao domicílio, habitações próximas à floresta primária, com a ocorrência de leishmaniose tegumentar americana em crianças pequenas, sugerindo transmissão no intra e no peridomicílio, em alguns casos, crianças penetrando na floresta.

Leishmaniose; Criança; Infecção nais - Assentamento do Iporar e Assentamento Tarumã Mirim - contribuem com a maior casuística. O período de maior registro de casos é nos meses de novembro a abril em razão da maior pluviosidade, quando também ocorre aumento das populações e dos transmissores 11,13.

No período de janeiro de 1991 a julho de 2000, surgiram no Município de Manaus 41 novos focos de transmissão de leishmaniose tegumentar americana, distribuídos entre invasões ou ocupações desordenadas, novos conjuntos residenciais, áreas de lazer e projetos agropecuários? fato que também contribui para maior exposição das crianças.

Os resultados encontrados em crianças diferem dos que vêm sendo observados nos últimos anos, para todas as faixas etárias, com grande parte dos registros de ocorrência na periferia de Manaus. Tais dados demonstram que a grande maioria dos casos era procedente de dois assentamentos populacionais agrícolas (Ramal do Iporar, no Município de Rio Preto da Eva e Assentamento Tarumã Mirim, no Município de Manaus). Permanece o padrão de transmissão relacionado a habitações próximas à floresta primária e atividades de manejo florestal, mas a ocorrência de leishmaniose tegumentar americana em crianças, especialmente as muito pequenas, com ou sem história de penetração na mata, sugerem transmissão no intra e no peridomicílio.

\section{Colaboradores}

J. A. O. Guerra coordenou e participou de todo o trabalho de pesquisa e elaboração e redação final de todo o texto. M. G. V. Barbosa participou da pesquisa, da discussão do trabalho e da analise estatística. A. C. S. P. Loureiro participou do trabalho de pesquisa, da elaboração e do atendimento aos doentes e da revisão bibliográfica inicial. C. P. Coelho participou do atendimento aos doentes, tabulação e análise dos dados, redação do manuscrito na pesquisa bibliográfica. G. G. Rosa participou do atendimento aos doentes, redação do manuscrito e da pesquisa bibliográfica. L. I. A. C. R. Coelho participou da parte de laboratório na pesquisa e da elaboração do texto. 


\section{Referências}

1. Special Programme for Research and Training in Tropical Disease. Report on leishmaniasis. Geneva: World Health Organization; 2004. (Scientific Working Group).

2. Fundação Nacional de Saúde. Manual de controle da leishmaniose tegumentar americana. Brasília: Ministério da Saúde; 2000.

3. Barret TV, Senra MS. Leishmaniasis in Manaus, Brazil. Parasitol Today 1989; 5:255-7.

4. Lainson R. Our present knowledge of the ecology and control of leishmaniasis in the Amazon Region of Brazil. Rev Soc Bras Med Trop 1985; 18:47-56.

5. Talhari S, Arias JA, Cunha MGS, Naiff RD, Freitas RA, Barrett T. Leishmaniose no Estado do Amazonas - aspectos epidemiológicos clínicos e terapêuticos. An Bras Dermatol 1988; 63:433-8.

6. Guerra JAO, Souza AS, Lima AA, Dias CMF, Guerra MVF. Leishmaniose tegumentar americana (LTA) - avaliação de dois anos de trabalhos com reservatórios em área periférica da cidade de Manaus. Rev Soc Bras Med Trop 2001; 34 Suppl 1:220.

7. Paes MG. Estudo de quatro espécies de Lutzomyia França, 1924 (Diptera, Psychodidae) em área endêmica de leishmaniose tegumentar americana na periferia de Manaus [Dissertação de Mestrado]. Manaus: Instituto Nacional de Pesquisas da Amazônia/Fundação Universidade do Amazonas; 1991.

8. Paes MG, Fé NF, Coelho LIARC, Oliveira RS, Sousa JU, Teixeira MR, et al. Sobre a ocorrência de leishmaniose tegumentar em rua de bairro de implantação antiga na cidade de Manaus (AM). Rev Soc Bras Med Trop 1998; 31 Suppl 1:97.

9. Andrade SL. Leishmaniose tegumentar americana em área de ocupação recente na periferia da cidade de Manaus Estado do Amazonas Brasil [Dissertação de Mestrado]. Rio de Janeiro: Departamento de Medicina Tropical, Instituto Oswaldo Cruz; 1997.

10. Paes MG, Barros MLB, Toledo LM. Considerações sobre a produção da Leishmaniose tegumentar americana no Estado do Amazonas In: IñiguezRojas LB, Toledo LM, organizadores. Espaço e doença: um olhar sobre o Amazonas. Atlas de geografia médica. Rio de Janeiro: Editora Fiocruz; 1998. p. 105-13.

11. Guerra JAO, Barros MLB, Guerra MVF, Talhari S, Paes MG. Leishmaniose tegumentar no município de Manaus - aspectos epidemiológicos. Rev Soc Bras Med Trop 1998; 31 Suppl 1:72.

14. Brandão Filho S, Campbell-Lendrum D, Brito MEF, Shaw JJ, Davies CR. Epidemiological surveys confirm na incrasing burden of cutaneous leishmaniasis in north-east Brazil. Trans Royal Soc Trop Med Hyg 1999; 93:488-94.

12. Castro EA, Soccol VT, Membrive N, Luz E. Estudo das características epidemiológicas e clínicas de 332 casos de leishmaniose tegumentar notificados na região norte do Estado do Paraná de 1993 a 1998. Rev Soc Bras Med Trop 2002; 35:445-52.
13. Ueslei T, Kühl JB, Rodrigues M, Santos ES, Santos DR, Maróstica LMF. Flebotomíneos coletados em matas remanescentes e abrigos de animais silvestres de zoológico no perímetro urbano de Maringá, sul do Brasil. Estudo preliminar. Rev Soc Bras Med Trop 1998; 31:517-22.

15. Forattini OP, Rabello EX, Serra OP, Cotrim MG, Galati EA, Barata JM. Observações sobre a transmissão da leishmaniose tegumentar no Estado de São Paulo, Brasil. Rev Saúde Pública 1976; 10:3143.

16. Ampuero J, Urdaneta M, Macêdo VO. Factores de riesgo para la transmisión de leishmaniasis cutánea en niños de 0 a 5 años en un área endémica de Leishmania (Viannia) braziliensis. Cad Saúde Pública 2005; 21:161-70.

17. Guerra JAO, Coelho LIARC, Cabral EG, Teixeira MRA, Moura MA, Paes MG. Comparação entre métodos para coleta de amostra de sangue em inquérito sorológico para leishmaniose em cães procedentes de áreas de transmissão e não transmissão humana de leishmaniose tegumentar americana no município de Manaus. NewsLab 2002; 55:7280.

18. Davies CR, Reithinger R, Campbell-Lendrum D, Feliciangeli D, Borges R, Rodriguez N. The epidemiology and control of leishmaniasis in Andean countries. Cad Saúde Pública 2000; 16:925-50.

19. Costa JML, Balby ITA, Rocha EJS, Silva JM, Rebelo M, Ferreira LA, et al. Estudo comparativo da leishmaniose tegumentar americana em crianças e adolescentes procedentes das áreas endêmicas de Buriticupu (Maranhão) e Corte de Pedra (Bahia), Brasil. Rev Soc Bras Med Trop 1998; 31:279-88.

20. Martins LM, Rebelo JMM, Santos MCF, Costa JML, Silva AR, Ferreira LA. Ecoepidemiologia da leishmaniose tegumentar no Município de Buriticupu, Amazônia do Maranhão, Brasil, 1996 a 1998. Cad Saúde Pública 2004; 20:735-43.

21. Passos VMA, Falcão AL, Marzochi MCA, Gontijo CMF, Dias ES, Barbosa-Santos EGO, et al. Epidemiological aspects of american cutaneous leishmaniasis in a periurban area of the metropolitan region of Belo Horizonte, Minas Gerais, Brazil. Mem Inst Oswaldo Cruz 1993; 88:103-10.

22. Natal SS, Viana AB, Cordeiro JA, Cavasini CA. American cutaneous leishmaniasis in the State of Acre, Brazil. Rev Saúde Pública 1999; 33:554-9.

23. Silveira FT, Ishikawa EA, De Souza AAA, Lainson R. An outbreak of cutaneous leishmaniasis among soldiers in Belém, Pará State, Brazil caused by Leishmania (Viannia) lindenbergi n. sp., a new leishmanial parasite of man in the Amazon region. Parasite 2002; 9:43-50.

24. Sosa-Estani S, Segura EL, Gomez A, Salomón OD, Peralta M, Coutada V, et al. Leishmaniose cutânea no Norte da Argentina: fatores de risco identificados num estudo caso-coorte em três municípios de Salta. Rev Soc Bras Med Trop 2001; 34:511-7. 
25. Campbell-Lendrum D, Dujardin JP, Martinez E, Feliciangeli D, Perez JE, Silans L, et al. Domestic and peridomestic transmission of American cutaneous leishmaniasis: changing epidemiological patterns present new control opportunities. Mem Inst Oswaldo Cruz 2001; 96:159-62.

26. Guerra JAO, Talhari S, Paes MG, Garrido M, Talhari JM. Aspectos diagnósticos da leishmaniose tegumentar americana em militares simultaneamente expostos à infecção na Amazônia. Rev Soc Bras Med Trop 2003; 36:587-90.
27. Arias JR, Naiff RD, Miles MA, Souza AA. The opossum Didelphis marsupialis (Marsupialia: Didelphidae) as a reservoir host of Leishmania brasiliensis guyanensis in the Amazon Basin of Brazil. Trans $\mathrm{R}$ Soc Trop Med Hyg 1981; 75:537-41.

28. Guerra JAO, Ribeiro JAS, Coelho LIARC, Barbosa MGVB, Paes MG. Epidemiologia da leishmaniose tegumentar na Comunidade São João, Manaus, Amazonas, Brasil. Cad Saúde Pública 2006; 22:2319-27.

29. Pinto PL, Cabral PH, Meneghinni T, Santos V, Haad N, Moraes T, et al. Processos de ocupação e atividades humanas no assentamento Tarumã mirim, (BR 174 km 21, Manaus) e sua relação com a produção e manutenção de endemias - uma análise crítica. Rev Soc Bras Med Trop 2005; 36 Suppl 1:279.

Recebido em 13/Jul/2006

Versão final reapresentada em 08/Mar/2007

Aprovado em 30/Mar/2007 